Session 2793

\title{
Interactive Tutorial for an Introductory Electrical Engineering Course
}

\author{
Maurice F. Aburdene, Rami W. Zarrouk, Ryan S. Magargle \\ Bucknell University
}

\begin{abstract}
This paper presents a tutorial and diagnostic tool called, "What You Already Know." The objective of this tutorial is to prepare students for their first electrical engineering course, and it is designed to diagnose what the students already know and aid them in understanding some basic concepts through hands-on experience. The tutorial is computer-based and utilizes links between Microsoft Word and Excel to produce interactive graphs that can be modified by the student. Another version of this tutorial is available using Mathcad, a tool that allows the student to integrate text, mathematical computation, and graphics into a single worksheet.
\end{abstract}

Students use the tutorial to assess their ability to visualize plots of sinusoids and exponentials in various mathematical forms. In addition, the tutorial checks student understanding of differentiation and integration of various waveforms. Other topics include simple design and optimization of single variable systems, and the matrix representation and solution of linear equations. In addition, the tutorial covers binary number systems and weighted sums. Students are asked to find the average and variance of sinusoidal signals and randomly selected sample values of resistors. The exercises were developed to help the instructor perform an "academic checkup" or "background check" of students taking their first course in electrical engineering during their second semester of the first year. However, it has been used as a diagnostic tool for other classes as well.

\section{Introduction}

Students having a strong background in the first year of electrical engineering tend to have a distinct advantage in higher level courses. In the past, standard paper-and-pencil methods such as homework and lecturing relied heavily on the visualization capabilities and material absorption rate of the student. Newer interactive methods, using computer technology, reduce the effort and time needed to understand new concepts. It is still important for the professor to teach the concepts in a classroom setting, but these interactive tools help the student increase his or her understanding of the problems explained in class. This increased understanding comes after the student has fully explored the problems taught in class using interactive tools. These interactive tools will allow the student to modify certain parameters in a problem and see the corresponding output.

The combination of Microsoft (MS) Word and Excel has been used because these programs are usually packaged together and can be linked to interactively modify graphs and equations. Mathcad has also been used because it is inherently interactive and because of the ability to produce animation movies, which can help the student better understand the problem. In addition, the use of these programs will further the student's expertise with the above software packages and computers in general. 
The ability to visualize data in graphical form is a fundamental tool that will help the introductory level student develop an intuitive feel for many electrical engineering concepts. With regard to graphical data, the exponential decay and sinusoidal waveform are two very common electrical engineering responses. The student is presented with a list of parameters that can be modified, such as the exponent parameter, $\alpha$, shown in Figure 1.

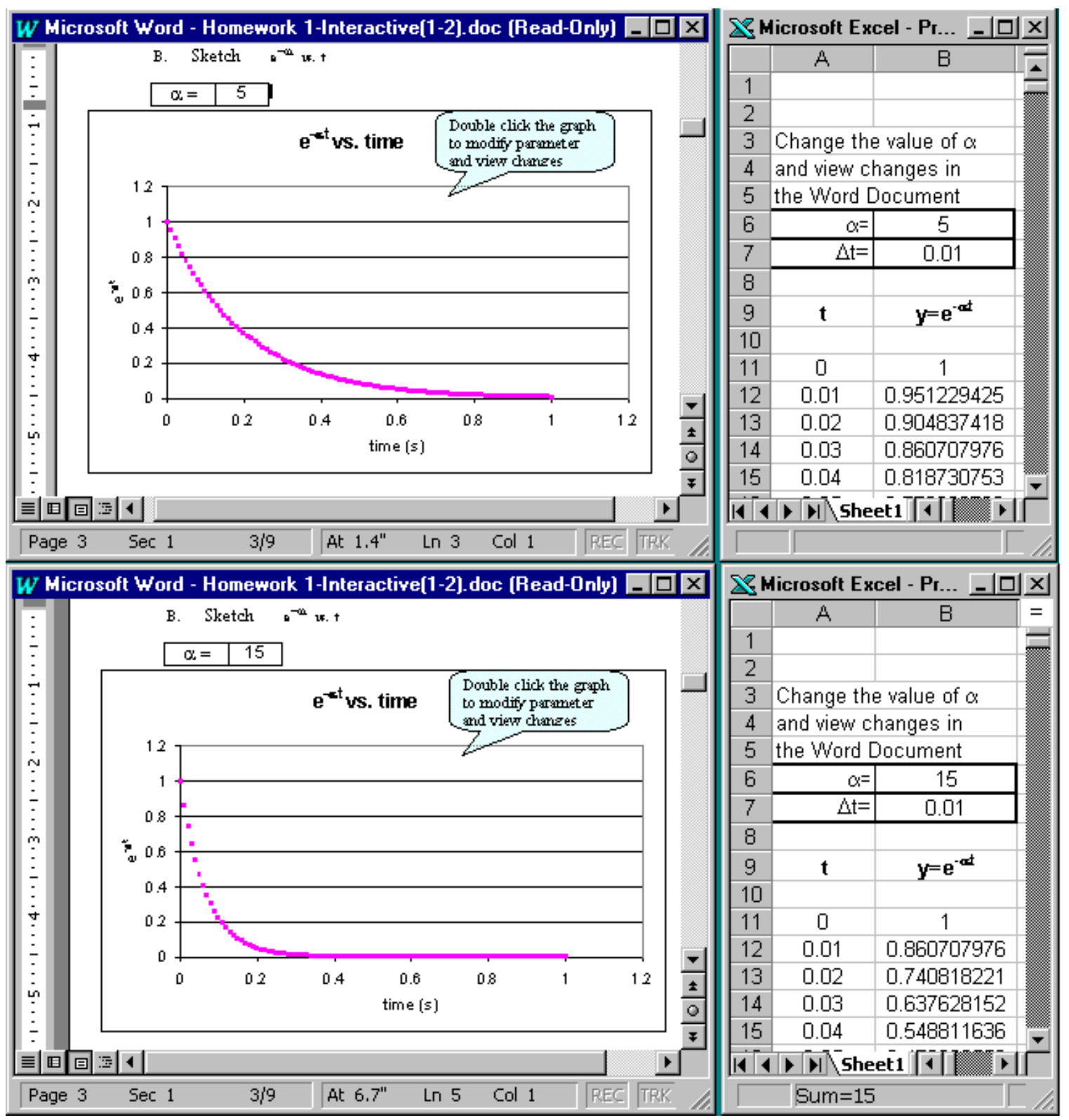

Figure 1. Interaction between Microsoft Word and Excel. A user changes the $\alpha$ value in Excel and observes the result in Word. The top figure shows $\mathrm{e}^{-5 t}$ vs. $t$, whereas the bottom figure shows $\mathrm{e}^{-15 t}$ vs. $\mathrm{t}$. 
The student interacts with the tutorial by double clicking on the graph in MS Word, which opens the corresponding Excel worksheet. In Excel, one would be able to see some calculations that are dependent on a list of variables, which a user is able to modify to see changes in Word. In Figure 2 , the value of the exponent $(\alpha)$ was changed from 5 to 15 , and the corresponding output is seen in MS Word. It is conceivable that one can do the whole tutorial in MS Excel; however, students can add documentation in MS word to increase their understanding of the material.

\section{Differentiation}

Differentiation is an important concept without which students seem to struggle in higher-level engineering courses. With an interactive method, students will be able to enter a function and see its corresponding derivative almost instantly. As an example, consider the triangular function written in Mathcad notation and plotted in Figure 2.

$\mathrm{v}(\mathrm{t}):=\mathrm{r}(\mathrm{t}) \cdot \mathrm{u}(1-\mathrm{t})+\mathrm{r}(\mathrm{t}-1) \cdot \mathrm{u}(2-\mathrm{t})$ where $\mathrm{u}(\mathrm{t})$ is the step function and $\mathrm{r}(\mathrm{t})$ is the ramp function.

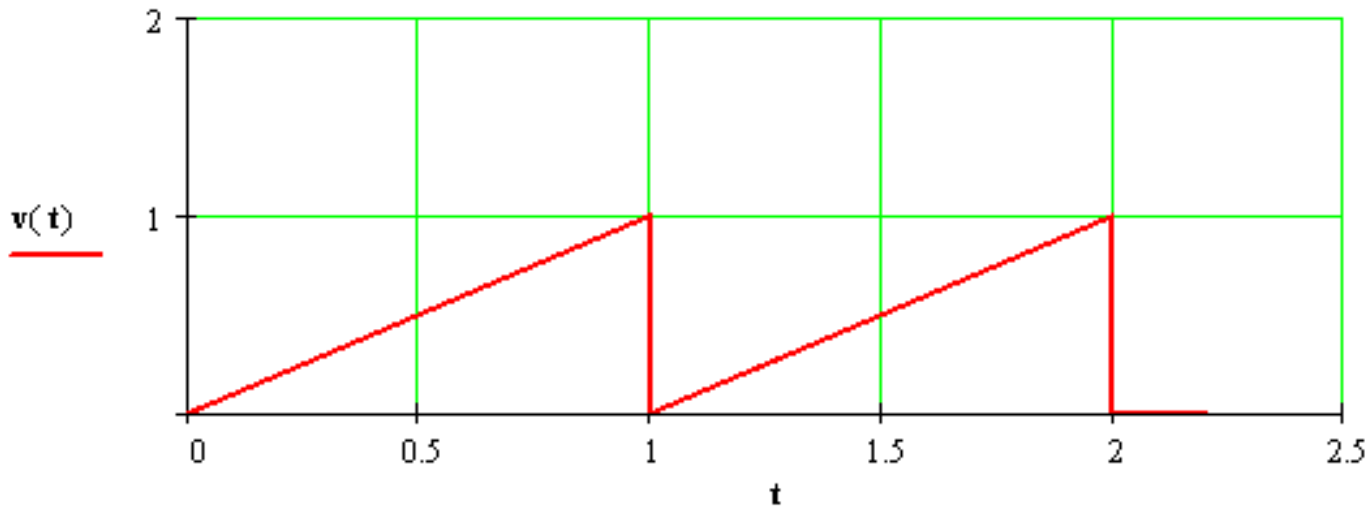

Figure 2. Mathcad plot of v(t) vs. $t$

Once a user enters a function for $v(t)$, a derivative will be found and plotted. Figure 3 shows the derivative of v(t) vs. t.

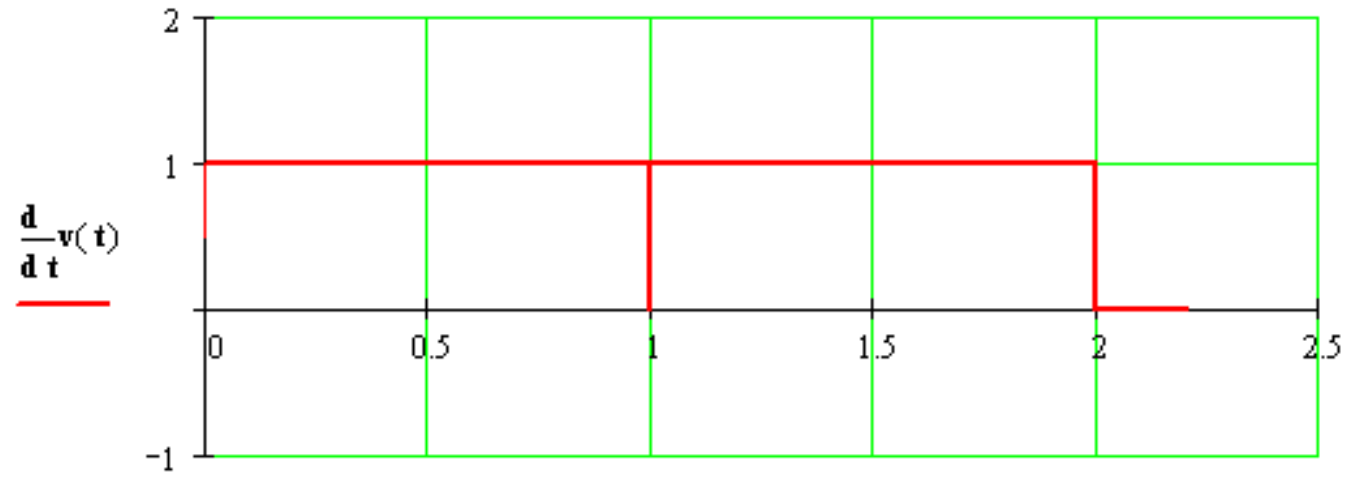

Figure 3. Mathcad Plot of the derivative of $v(t)$ vs. $t$ 


\section{Integration}

The concept of integration is as important as differentiation for engineers. From convolution to finding the root mean square value of a signal, integration is no doubt a must-know operator. As an example, consider the function where $\prod(t)$ is the unit pulse function.

$$
\mathrm{i}(\mathrm{t}):=\Pi(\mathrm{t}-0.5)+\Pi(\mathrm{t}-2.5)
$$

If $y(t):=\int_{0}^{t} i(\tau) d \tau$ then a plot of both $i(t)$ and $y(t)$ is shown in Figure 4 .

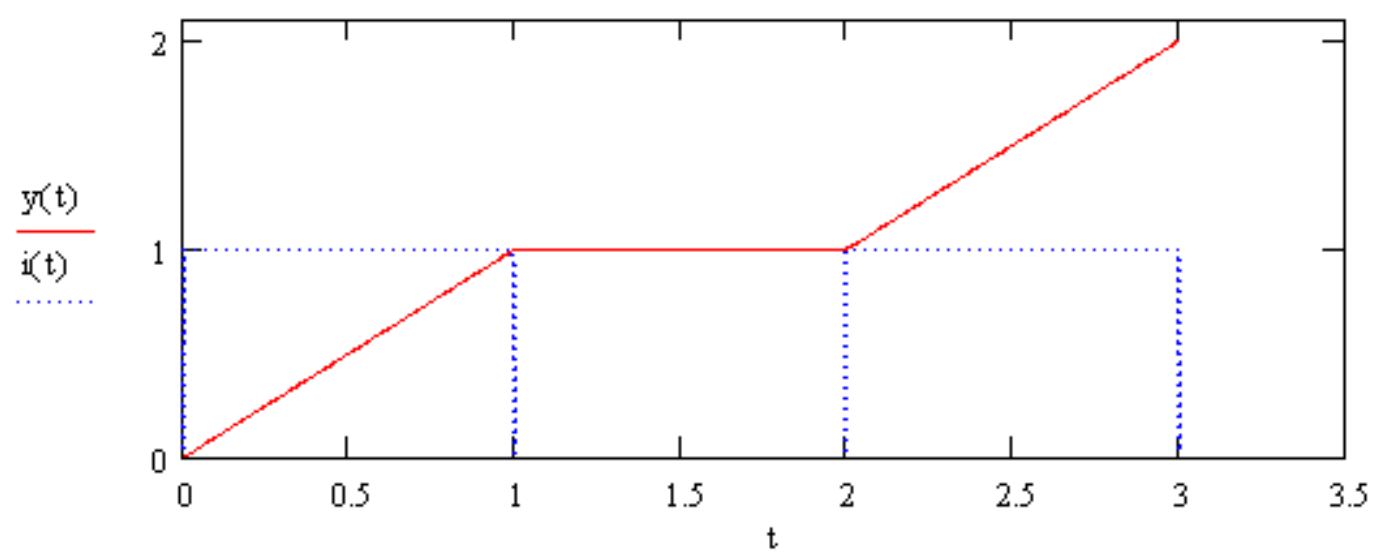

Figure 4. Plot of both $\mathrm{i}(\mathrm{t})$ and its integral $\mathrm{y}(\mathrm{t})$. The continuous red line is the integral whereas the dotted blue line is the input signal. The input $\mathrm{i}(\mathrm{t})$ is zero between 1 and 2 .

\section{Single variable systems}

So many times, a student encounters systems in which he or she has to change a variable and note the output. One particular example is when one needs to change a resistor value in an analog circuit and observe the resultant output. A student in an introductory course has to be able to graph the independent variable versus the dependent variable, and then relate the graph to a real life application. For an example, consider a voltage, V, that depends on two resistor values, R0 and R. Furthermore, suppose you are solely interested in finding out how only one resistor value impacts the output voltage. The describing system equations expressed in Mathcad notation are:

$$
\mathrm{R}:=10 \quad \mathrm{R} 0:=0 . .100 \quad \mathrm{~V}(\mathrm{R}, \mathrm{R} 0):=\frac{\mathrm{R}}{\mathrm{R}+\mathrm{R} 0}
$$

where R is held constant at $10 \Omega$ and R0 varies from 0 to $100 \Omega$ in increments of $1 \Omega$. Figure 5 shows how the voltage will vary according to the above system equation. 


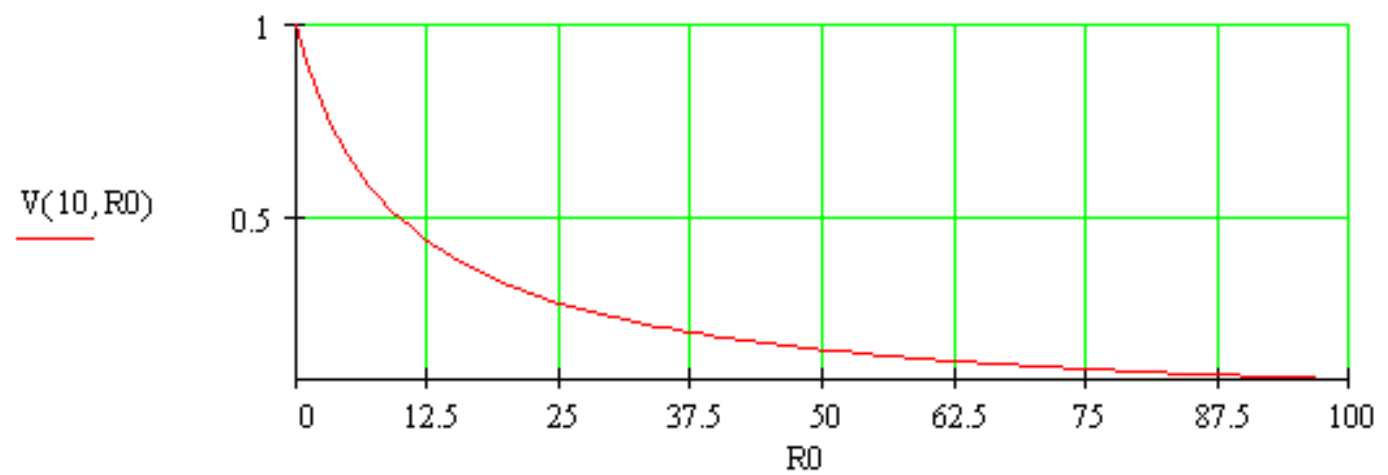

Figure 5. A plot of the output voltage as a function of R0.

Average and rms value of a periodic signal

The root mean square (rms) value is a relatively easy concept that is sometimes perceived as difficult by a student newly introduced to an introductory electrical engineering course. In fact, some students might have a vague idea of the difference between the average value and the rms value of a signal. In addition, professors in higher-level courses take it for granted that a student knows what an effective current/voltage or rms current/voltage means; therefore, professors in introductory courses need to make sure that their students fully understand the concept. One effective way of embedding that concept in the students' minds is by providing an interactive environment in which a student can change the periodic signal and observe the square of the signal, the mean of the square, and the root of the mean of the square (rms). A sinusoid is a typical example for finding the rms value.

Consider $\quad v(t):=3 \cdot \sin (2 \cdot \pi \cdot f \cdot t)$

where $\mathrm{t}$ varies from 0 to $2 / 60$ seconds in increments of 0.0001 second, and $\mathrm{f}$ is $60 \mathrm{~Hz}$.

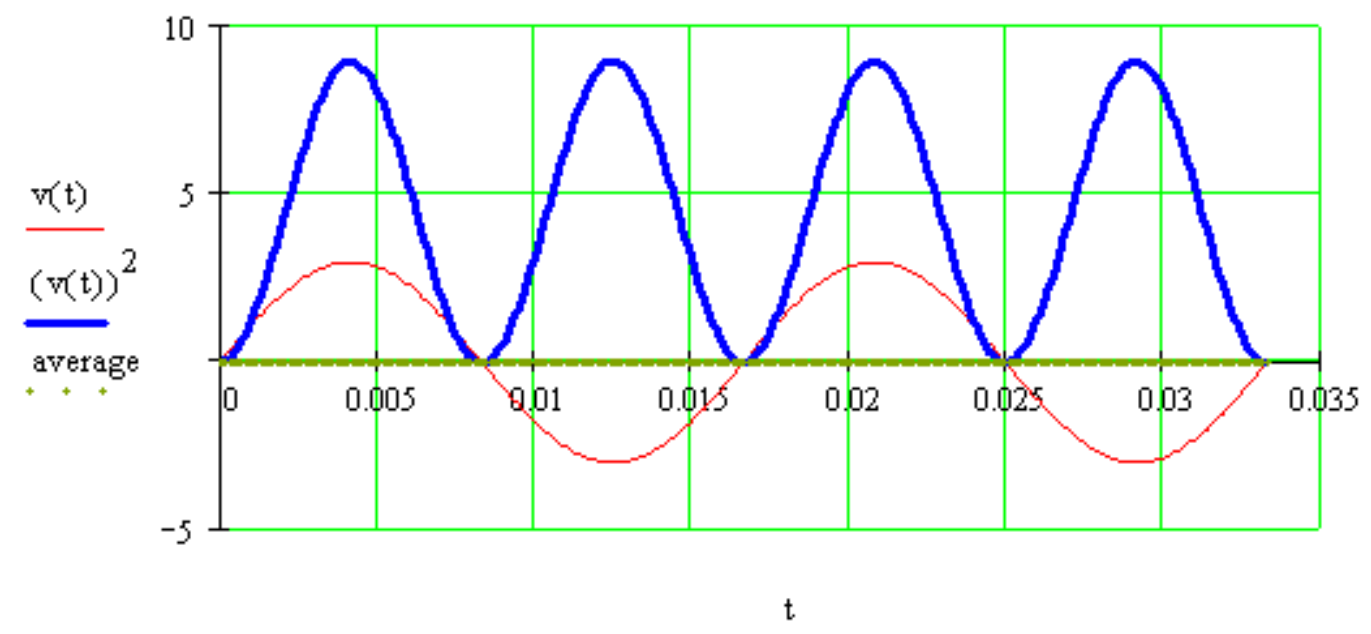

Figure 6. Plot of $v(t)$ vs. $t$ shown in thin red line, average of $v(t)$ is shown in brown dots (zero in this case), and $\mathrm{v}(\mathrm{t})^{2}$ is shown in thick blue line. 


$$
\begin{array}{lc}
\text { Average_of_Square }=\frac{1}{\mathrm{~T}} \cdot \int_{0}^{\mathrm{T}} \mathrm{v}(\mathrm{t})^{2} \mathrm{dt} & \text { Average_of_Square }=4.5 \\
\text { RMS }:=\sqrt{\text { Average_of_Square }} & \text { RMS }=2.121
\end{array}
$$

A user will be able to see the newly calculated value for the RMS upon entering a new periodic signal

\section{Conclusion}

Students at Bucknell University have shown enthusiasm about the tutorial, which they thought increased their understanding of the fundamental material. The students' justifications for this enthusiasm include the ease of use of this interactive method, the importance of the concepts used in the tutorial, and the variety of the problems included. In the tutorial, aside from the problems presented in this paper, other problems that were considered include the solution of 2variable simultaneous equations, conversion of binary-coded decimal to decimal equivalence, manipulation of matrices, and demonstration of statistical concepts such as finding the mean, the variance, and the standard deviation.

\section{Acknowledgments}

Microsoft is a registered trademark of Microsoft Corporation. Mathcad is a trademark of Mathsoft incorporated.

\section{MAURICE FELIX ABURDENE}

Maurice F. Aburdene is the T. Jefferson Miers Professor of Electrical Engineering and Professor of Computer Science at Bucknell University. He has also taught at Swarthmore College, State University of New York at Oswego, and the University of Connecticut. Professor Aburdene was a project engineer and project manager at the Bristol Company, a visiting research scientist at MIT's Laboratory for Information and Decision Systems, and held various summer fellowships with NASA and the Naval Research Laboratory.

\section{RAMI WILLIAM ZARROUK}

Rami William Zarrouk is a graduate student in electrical engineering at Bucknell University. He has a Bachelor degree of electrical engineering from Christian Brothers University in Memphis, Tennessee. He is Tau Beta Pi and IEEE member. Rami is also a recipient of the 1998 Philip-Morgan Award for best engineering design. He is currently working as a teacher assistant for an introduction to digital systems class.

\section{RYAN SCOTT MAGARGLE}

Ryan Magargle is a junior in electrical engineering at Bucknell University. He is a member of IEEE, Tau Beta Pi, Alpha Lambda Delta, Phi Eta Sigma, and Alpha Phi Omega. He is also minoring in physics, economics, and mathematics. Ryan is a teacher assistant for physics and electrical engineering courses. 